\title{
Congenital hypothyroidism presenting with seizures in a newborn
}

Hany Aly, David E. Kanter, Susan A. Fisher-Owens

Department of Neonatology, The Children's National Medical Center

and the George Washington University Hospital Washington, U.S.A.

\begin{abstract}
Hypothyroidism is not known to present with seizures in neonates. This report describes a newborn who presented with seizures that were not controlled with several antiepileptic medications. The infant was later diagnosed with hypothyroidism, and started on Lthyroxine. Seizures subsequently ceased and the antiepileptic medications were stopped. (J Pediatr Neurol 2004; 2(2): 111-113).
\end{abstract}

Key words: seizures, congenital hypothyroidism.

\section{Introduction}

Several conditions are linked clinically with seizures in newborn infants. Some of these conditions are prenatal: such as intra-uterine ischemia and congenital viral infections; some conditions are perinatal: such as asphyxia and intracranial hemorrhage; or postnatal such as: hypoglycemia and congenital errors of metabolism. In this report, we describe a rare association of seizures with thyroid disturbances in a neonate. It seems to us that seizures represent a wide variety of diseases some of which are known, while others are yet to be discovered (1).

\section{Case Report}

A term infant was born to a 38-year-old mother whose prenatal history was significant for major depression requiring daily antidepressant therapy

Correspondence: Hany Aly, M.D.,

$90023^{\text {rd }}$ Street, NW Room G-132

Washington, Dc 20037, U.S.A.

Tel: (202) 715-5350, fax: (202) 715- 5354

E-mail: haly@mfa.gwu.edu

Received: October 27, 2003.

Revised: December 11, 2003.

Accepted: December 12, 2003. and gestational diabetes controlled by diet alone. She had a past history of hypothyroidism, but did not require any replacement therapy for 9 years prior to this delivery. Of note, the mother had gained little weight during pregnancy. She was colonized with group B streptococcus and had received one dose of ampicillin during labor.

Thick meconium was noted in the amniotic fluid and an amnio-infusion was commenced. Subsequently, a cesarean section was performed for a non-reassuring fetal heart rate and failure to progress. Maternal fever was noted prior to delivery. Apgar scores were 8 and 9 at 1 and 5 minutes respectively. Facial continuous positive airway pressure (CPAP) was initiated in the delivery room for respiratory distress, when grunting, flaring, and retractions were noted and continued on nasal CPAP after transfer to the neonatal intensive care unit.

Physical examination on admission showed a male neonate with a weight of $2750 \mathrm{~g}$ (10-25th percentile), length of $45 \mathrm{~cm}(<10$ th percentile), and head circumference of $34 \mathrm{~cm}$ (25-50th percentile). He had a prominent caput succedaneum. Mild to moderate subcostal retractions and grunting were noted while on CPAP. The rest of the physical exam was within normal limits. Color was pink and oxygen saturation was $95 \%$. A portable chest radiograph revealed clear lung fields. Respiratory distress resolved within a few hours and the infant was weaned off CPAP within the first day of life. Initial blood gases showed acidosis that resolved within 3 hours, but no hypoxemia. The infant was empirically treated with ampicillin and gentamicin for seven days.

On the 6th day of life, the infant was noted to have rhythmic, non-suppressible jerking motions that started in the right upper extremity and rapidly became generalized to involve all extremities. A course respiratory stridor accompanied the seizure activity. The seizure activity was unresponsive to an initial phenobarbital bolus. Only after controlled after total doses of $40 \mathrm{mg} / \mathrm{kg}$ of phenobarbital, 0.1 $\mathrm{mg} / \mathrm{kg}$ of lorazepam and $25 \mathrm{mg} / \mathrm{kg}$ of phenytoin was the seizure controlled. A lumbar puncture was 
Table 1. Thyroid function studies

\begin{tabular}{|c|c|c|c|c|c|}
\hline $\begin{array}{l}\text { Age } \\
\text { (day) }\end{array}$ & $\begin{array}{l}\text { Weight } \\
\text { (gram) }\end{array}$ & $\begin{array}{l}\text { L-Thyroxine } \\
\text { dose }(\mu \mathrm{g} / \text { day })\end{array}$ & $\begin{array}{l}\text { TSH } \\
(\mathrm{N}: 0.4-4.7 \mathrm{uIU} / \mathrm{mL})\end{array}$ & $\begin{array}{l}\text { Free T4 } \\
(\mathrm{N}: 0.7-1.8 \mathrm{ng} / \mathrm{dL})\end{array}$ & $\begin{array}{l}\text { Free T3 } \\
(\mathrm{N}: 1.5-3.5 \mathrm{pg} / \mathrm{mL})\end{array}$ \\
\hline 8 & 2845 & 0 & 0.25 & 0.5 & NP \\
\hline 9 & 2815 & 0 & 0.67 & 0.5 & 1.41 \\
\hline 15 & 2970 & 25 & 0.71 & 1.4 & NP \\
\hline 19 & 3075 & 25 & 0.55 & NP & NP \\
\hline
\end{tabular}

TSH: Thyroid stimulating hormone; T4: Thyroxine; T3: Triiodothyronine; NP: Not performed.

performed and cerebrospinal fluid examination was normal. The infant was started on maintenance doses of both phenobarbital and phenytoin. Post-ictally, he was noted to exhibit temperature instability and was placed under a radiant warmer for a better control of his temperature. Examination of serum electrolytes, blood glucose, serum amino acids and urine organic acids were within normal limits for age.

After resolution of the large head caput, it was noted that the infant had widened sutures and enlarged posterior fontanel. The posterior fontanel measured $4 \mathrm{~cm}$ at the widest diameter. Computed tomography $(\mathrm{CT})$ of the brain obtained within 24 hours of the initial seizure was not conclusive. An ophthalmologic examination two days after the initial seizure activity was also normal. Magnetic resonance imaging (MRI) of the brain did not reveal any abnormalities. An electroencephalogram (EEG) did not reveal patterns consistent with seizure activity. This EEG was performed while the patient was on both phenytoin and phenobarbital.

Newborn metabolic screen results were not available as of the 9th day of life. However, because of the infant's hoarse cry and wide posterior fontanel, thyroid function tests including triiodothyronine (T3), thyroid stimulating hormone (TSH) and free thyroxine (T4) were done. The results of these tests were abnormal. Thyroid tests were repeated and confirmed (Table 1). The infant was started on daily L-thyroxine $(12.5 \mu \mathrm{g})$ replacement therapy. Maternal TSH and T4 were found to be within normal limits. The infant had a few more episodes of seizure activity that were noticed before the next scheduled dose of L-thyroxine. The dose was increased to 25 $\mu \mathrm{g}$, and following this the infant remained free of further seizures. Antiepileptic medications were then discontinued and the infant remained stable. Because the infant did not have any other signs suggestive of pituitary insufficiency, no further work up for this was done.

A skeletal survey done on the 13th day of life was consistent with delayed bone age. The infant was successfully weaned to an open crib by the 15 th day of life and his temperature was well maintained. EEG was repeated on the 20th day of life, while off anti-epileptic medications, and remained normal for age. The infant was discharged home on day of life number 22. Follow up with a pediatrician and a pediatric endocrinologist was recommended.

The infant presented to the emergency room of the Children's Hospital with seizure activity at 2 months of age. Similar work up revealed hypothyroidism and the dose of L-thyroxine was increased. The infant continued on L-thyroxine without any seizure activity. Neurological evaluation by the primary care pediatrician was normal at 6 months of age.

\section{Discussion}

This infant initially presented with seizures and was also diagnosed with congenital hypothyroidism. Seizures in the newborn period may be a manifestation of hypoxic-ischemic encephalopathy (HIE), metabolic diseases, intracranial hemorrhage, infections, cerebral dysgenesis, hypoglycemia and hypocalcemia (1). Hypoxic-ischemic encephalopathy would seem to be the likely cause for seizures in an infant who had respiratory distress, meconium at delivery and acidosis shortly after birth. However, this infant did not have hypoxemia, had a normal neurological evaluation as well as normal head imaging. The onset of seizure activity was not consistent with the diagnosis of HIE. Seizures thought to be caused by HIE typically present within the first 72 hours of life (1). Other etiologies such as cerebral dysgenesis and late onset hypocalcemia would fit this infant's timing of onset, but laboratory data and imaging studies did not support either diagnosis.

Although the association of hypothyroidism and seizures is unusual, this infant presented with many signs that strengthened the belief in this association. The low TSH and T4, the widened sutures with a large posterior fontanel, temperature instability, a hoarse cry and the delayed bone age are all consistent with this disease process. Because of the delayed bone age, hypothyroidism was believed to be congenital in origin. It is unlikely that hypothyroidism was caused by perinatal or postnatal injury, as there were no physical, laboratory or imaging signs of hypoxia. Neither a perinatal nor a postnatal event 
could explain the delayed bone age.

There were several mitigating circumstances in this infant, such as the presence of maternal gestational diabetes, maternal depression and the use of antidepressants. However, the likelihood of an accidental association between hypothyroidism and seizures in this infant is low. Seizures were only minimally responsive to anti-epileptic medications and resolved after thyroid replacement therapy was initiated. Also, the occurrence of the later "breakthrough" seizures in the neonatal intensive care unit was prior to the next scheduled dose of L-thyroxine doses, when the serum level of T4 was low. Seizures did not resume either clinically or on EEG with the cessation of anti-epileptic medications.

While classically hypothyroidism is associated with low T4 and high TSH, 1/100,000 newborns with congenital hypothyroidism are born with low $\mathrm{T} 4$ and normal TSH (2). These children may be missed by the neonatal metabolic screening (NMS) (3). TSH gradually climbs over the first few weeks of life. Of note, the routine NMS of this infant was normal. Alternatively, this infant may have hypothyroidism of central origin that transiently caused a low level of TSH at initial assessment. Finally, there could be another unexplored cause of seizures that was ameliorated with the use of L-thyroxine. This last hypothesis is supported by a previous report demonstrating a lower incidence of febrile seizures in hypothyroid infants while receiving L-thyroxine (4).

In conclusion, this appears to be a case of hypothyroid-associated seizure. There have been several published articles on the increased neuroexcitability of hypothyroid rats, but few cases in pediatric literature mention hypothyroidism and seizures (5). The purpose of this report is two-folds: to encourage pediatrician to investigate for hypothyroidism when clinically suspected, even in the face of a normal neonatal metabolic screening; and to encourage those in the midst of an unproductive neonatal seizure work-up to include thyroid studies if clinical signs and symptoms are sufficient to support the diagnosis.

\section{References}

1. Hill A, Volpe J. Neonatal seizures. In: Avery GB, Fletcher MA, MacDonald MG (eds). Neonatology: Pathophysiology and Management of the Newborn $\left(5^{\text {th }}\right.$ ed). Philadelphia: Lippincott Williams \& Wilkins, 1999, pp 1232-1234.

2. American Academy of Pediatrics. Newborn screening for congenital hypothyroidism: recommended guidelines. Pediatrics 1993; 91: 12031208.

3. Huang YC, Huang FY, Tsai TC, Hsu CH. Congenital hypothyroidism missed on newborn screening: report of two cases. Zhonghua Min Guo Xiao Er Ke Yi Xue Hui Za Zhi 1990; 31: 336-339 (in Chinese).

4. Asami T, Sasagawa KS, Kyo S, Asami K, Uchiyama M. Incidence of febrile convulsions in children with congenital hypothyroidism. Acta Paediatr 1998; 87: 623-626.

5. al-Qudah AA. Screening for congenital hypothyroidism in cognitively delayed children. Ann Trop Paediatr 1998; 18: 285-288. 